\title{
Terrestrial Planet Finder Coronagraph Observatory Summary
}

\author{
Virginia Ford ${ }^{1}$, Marie Levine-West ${ }^{1}$, Andy Kissil ${ }^{1}$, Eug Kwack ${ }^{1}$, \\ Tim Ho ${ }^{1}$, Phil Dumont ${ }^{1}$, Doug Lisman ${ }^{1}$, Peter Feher ${ }^{1}$, \\ and Terry Cafferty ${ }^{2}$ \\ ${ }^{1}$ Jet Propulsion Laboratory, California Institute of Technology, Pasadena, CA 91109, USA \\ email: Virginia.G.Ford@jpl.nasa.gov, Marie.B.Levine@jpl.nasa.gov, \\ Andrew.Kissil@jpl.nasa.gov, Eug-Yun.Kwack@jpl.nasa.gov, Timothy.Y.Ho@jpl.nasa.gov, \\ Philip.J.Dumont@jpl.nasa.gov, P.D.Lisman@jpl.nasa.gov, \\ ${ }^{2} \mathrm{TC}$ Technology
}

\begin{abstract}
Creating an optical space telescope observatory capable of detecting and characterizing light from extra-solar terrestrial planets poses technical challenges related to extreme wavefront stability. The Terrestrial Planet Finder Coronagraph design team has been developing an observatory based on trade studies, modeling and analysis that has guided us towards design choices to enable this challenging mission. This paper will describe the current flight baseline design of the observatory and the trade studies that have been performed. The modeling and analysis of this design will be described including predicted performance and the tasks yet to be done.
\end{abstract}

\section{Introduction}

NASA is funding a mission called Terrestrial Planet Finder (TPF) that intends to find and characterize terrestrial (or rocky) planets that might harbor life. This mission will look for Earth-like planets around nearby stars in the zone where liquid water could be present on the planet surface. In order to meet this criterion, the planets must be orbiting in the spherical region around a star called the habitable zone where water will be liquid.

When a planet is found in this zone, by studying the light from that planet, the presence of life can be detected from the spectrum of gases that are present in its atmosphere. The presence of life-indicating gases such as water, oxygen, ozone, carbon dioxide, nitrous oxide, methane, and chlorophyll in land plants can be detected in the spectrum of light reflecting off of a planet's atmosphere. The challenge of this mission is to suppress the parent starlight adequately to detect and characterize the fainter light from the planet.

In order to perform this mission, two instruments are being proposed: a visible coronagraph and an infrared interferometer - each with different methods of suppressing starlight. The coronagraph, called Terrestrial Planet Finder Coronagraph (TPF-C), shown in Figure 1, will be the focus of this report. Both instruments provide complementary data in different optical wavebands to establish the presence of life on any planets that may be found and studied.

\section{Technology Research and Development}

The most challenging technology areas needing development for this project are related to optical wavefront accuracy. Extreme accuracy is needed in order to block out the parent starlight adequately so that the orbiting planet can be detected. In the visible wavelength range of TPF-C, the light from the planet is reflected from its parent star and 


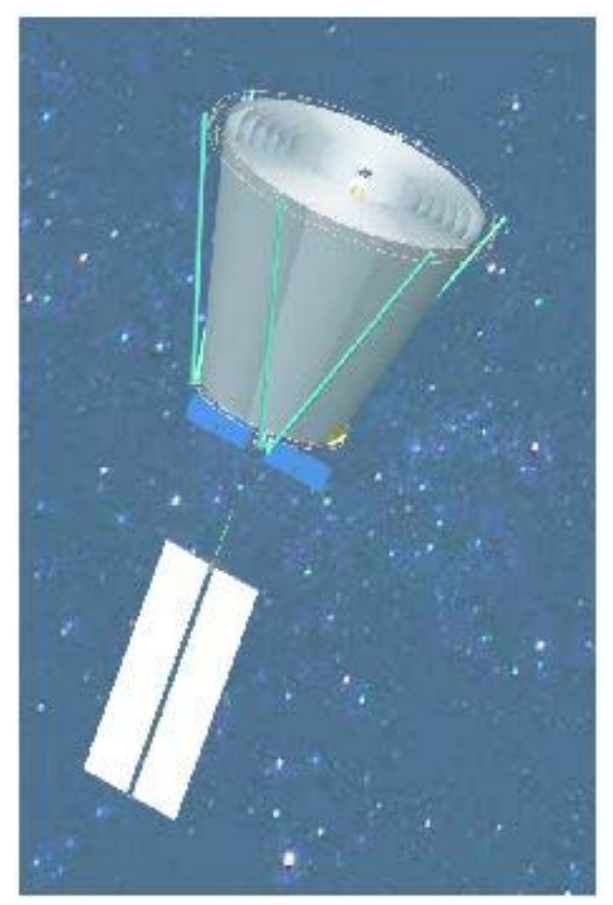

Figure 1. Terrestrial Planet Finder Coronagrapher-Flight Baseline 1 Configuration (Ho).

is 1010 times fainter than the parent star. This intensity reduction factor of 10 billion times requires a deeper understanding of light propagation physics including the effect of optical components on polarization, diffraction, phase, amplitude and wavelength and the crosstalk between these parameters. Test beds, component fabrication, measurements and modeling developments form the core of the technology development efforts that are underway.

Currently, the most mature test bed is called the High Contrast Imaging Testbed (HCIT). It is described in detail in the previous paper in this conference (Trauger 2005); but, in summary, consists of a large, isolated vacuum chamber containing an optical bench with a Lyot-style coronagraph. In addition, there are arrangements to test alternative coronagraph components and systems. The star-simulating source consists of a laser or white-light fed fiber-optic. Also in the system are a deformable mirror, and a camera. To date, this test bed has created a half-dark hole formed using only one DM to correct both phase and amplitude. An average contrast of $8 \times 10^{-10}$ has been attained that is repeatable and stable in the half area from $4 \lambda / D$ to $10 \lambda / D$; but in the half area from $4 \lambda / D$ to $5 \lambda / D$, the testbed has only achieved a contrast of $2 \times 10^{-9}$. The team is working to drive down the contrast in that critical smaller area.

Several areas of study support the HCIT and the TPF-C system design by theorizing, modeling, fabricating, and measuring characteristics of components in the HCIT. The goal is to measure component properties and model accurately the optical propagation effects of these components. The model will be validated using HCIT performance; then will be used to explore the best methods of starlight suppression for TPF-C. Some results are presented in a poster in Session 1 (Balasubramanian 2005) of this conference. There is a coordinated effort involving laboratories, NASA centers and universities that has led to increased understanding of electro-magnetic wave propagation through coronagraph components, and has given us a more accurate understanding of methods of suppressing 
diffracted and scattered starlight. Some of this work is represented in an upcoming paper in this conference (Kuchner 2005) and in a poster (Belikov 2005).

In addition to the technology studies related to attaining starlight suppression, a technology demonstration mirror (TDM) is being fabricated to demonstrate the surface quality, spatial frequency requirements, and light-weight construction required for the TPF-C primary mirror. Figure 2 shows some mirror core segments during fabrication. The TDM has a diameter, 1.8 meters, selected to represent the lowest spatial frequency of interest. Fabrication of the TDM will demonstrate that state-of-the-art technology can meet the spatial frequency requirements. Understanding mirror measurement and interpreting the measured data to ensure compliance with requirements also will be learned, along with materials and coating issues. Trade studies have resulted in the selection of lightweighted, fuse-bonded ULE as the substrate material. ITT and Corning are fabricating this mirror.

Alternative concepts for starlight suppression for this mission are also being studied. A pupil remapping study (Guyon 2005), a visible nulling concept (Shao 2005), and a comparison of coronagraph forms (Shaklan 2005) are presented orally and in posters in this conference.

\section{Observatory Design Status}

The current design is called the Flight Baseline 1 design concept. The mission and spacecraft choices for the FB1 design are shown in Table 1 and provide the frame work that defines the FB1 observatory requirements. These choices are not considered to be technology drivers - in fact, an attempt was made to stay within the limits of existing technology where possible so that resources at this phase of the project could focus more on areas that require technology development or non-heritage engineering.

The observatory is designed to examine stars in nearly the entire anti-sun semi hemisphere - a $5^{\circ}$ margin is included. The field of regard includes all stars located in

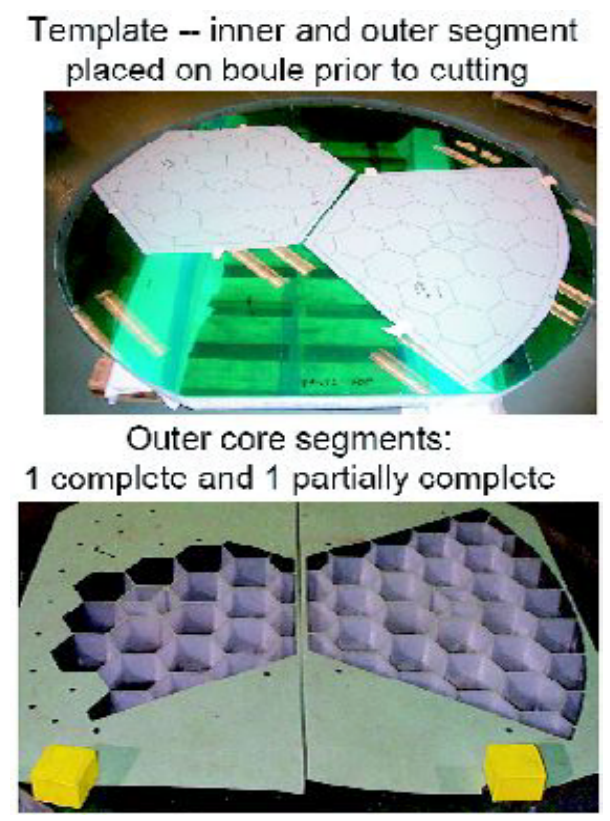

Figure 2. TDM core-made from 6 outer and one inner segment (Cohen, ITT). 


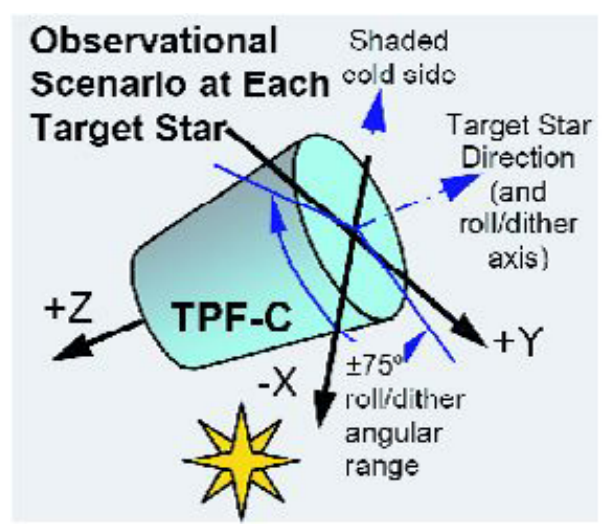

Figure 3. Observational scenario.

the cone defined as greater than or equal to $95^{\circ}$ away from the sun. As the observatory travels around the sun, this field-of-regard will sweep the entire sphere of the universe, allowing observation of all star targets of interest during nearly 5 months of the year. This field of regard provides the opportunity to view target stars multiple times during one year so that planets can be seen at a favorable position in their orbit-not behind or in front of the star.

During each star observation, the observatory will point at the chosen target star. Once the dynamics are stabilized, the observatory will collect light. Using adaptive optics, the wavefront errors will be reduced until the starlight is suppressed adequately and an image will be taken. Next the observatory will "dither" about its pointing axis by 30 degrees. Once the dynamics are stabilized, the observatory will take an image in this new position. This image will be subtracted from the previous image to eliminate residual light scattered from the observatory (which rolls with the dither). Any planets present would not roll and would be detectable. Because the primary mirror is oblong, it is most sensitive along its long axis. In order to completely study the habitable zone around a star, the long axis has to be rotated to positions that are $\pm 60^{\circ}$ away from the starting point. This is accomplished by a "roll" along the pointing axis. At each new roll position, the adaptive optics reset the wavefront and then the image gathering is repeated, including the dither. With $30^{\circ}$ dithers around three $60^{\circ}$ roll positions, the total angular rotation around the target direction axis is $\pm 75^{\circ}$ as shown in Figure 3 and 4. This observational scenario defines the thermal environmental perturbations that need to be investigated. The observatory must be stable enough between dither maneuvers to retain precise optical wavefront stability. After each roll, the wavefront correction is reset, so stability between roll positions is not as critical. Figure 5 shows a schematic of the design concept and Figure 6 shows the observatory design. The payload is surrounded by a large deployable conic-shaped v-groove sun shade which insulates the payload from the changing sun angles. The sunshade is structurally attached to the spacecraft through deployable arms and booms, so dynamic snaps or warping of the sunshade structures will be filtered through the spacecraft before reaching the sensitive payload.

The optical path enters the telescope baffle and reflects off an $8 \mathrm{~m} \times 3.5 \mathrm{~m}$ elliptical offaxis parabolic primary mirror. The light next reflects off the secondary mirror, towards a tertiary fold mirror that directs the beam into the coronagraph starlight suppression assembly. A pick-off mirror sends the outer portion of the beam to a general astrophysics instrument. 
Table 1. FB1 Mission \& Spacecraft Choices (Lisman \& Feher).

\begin{tabular}{|c|c|c|c|}
\hline & Parameter & Value & Comments \\
\hline \multirow{11}{*}{ 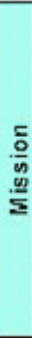 } & Durat on roquirod'goal & $5: 10$ ycare & Rosourcos tor 10 yoars \\
\hline & Orbit & 2 & Direct trajectory \\
\hline & Field of Regard & Sun ang es $>95^{\circ}$ & Potential earth'moon'planet constraints \\
\hline & Required AV & $65 \mathrm{~m}$ is & \\
\hline & Launch Erergy $\left(\mathrm{C}_{2}\right)$ & $-0.69 \mathrm{~km}^{2} \mathrm{j}^{2}$ & \\
\hline & Lanuch Vthicle & FEIV & \\
\hline & Launch Faring & 5 m diarnotor & imits orimary minco shorl axis lo $\sim 3.5 \mathrm{~m}$ \\
\hline & Launch Mass & $8200 \mathrm{~kg}$ & \\
\hline & Time to reach cperating orbit & 109 days & \\
\hline & Ground Station & $34 \mathrm{~m}$ DSN Ka Band & \\
\hline & Downlink Data Rate & EAfNlops & \\
\hline \multirow{9}{*}{ 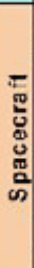 } & EOL Power & $3 \mathrm{kw}$ & provided by solar amays \\
\hline & Resaction Wheals & 6 Gondrict wheels & \\
\hline & Propelant & $242 \mathrm{~kg}$ Hydravins & \\
\hline & Thrusters & $1220 \mathrm{~N}$ & \\
\hline & Hi Rate Cownlink Frequency & Ke-Band & sug duration 2.5 rours per day \\
\hline & Encineriry Downlink Fiequency & X-Bend & \\
\hline & Uplink Frequenzy & X Band & \\
\hline & Transmitter Power & sowi & \\
\hline & Hi Grin Anlerına & $43 \mathrm{~d} / 8$ & D. 5 m palch antay \\
\hline
\end{tabular}

The primary mirror assembly is mounted semi-kinematically with a hexapod to an aft metering structure (AMS). Behind the primary mirror, the AMS supports thermal control heaters and the secondary mirror assembly deployable tower and the tertiary mirror assembly. Laser sources for laser metrology between the primary mirror and the second mirror are also mounted on the AMS. The laser metrology system will provide continuous feedback on the secondary mirror position. This will be used to adjust thermally-induced relative motions between the primary mirror and the secondary mirror. The aft metering structure mounts to a payload support structure (PSS) through three bi-pods. Further description of the telescope assembly is given in a poster (Ohl 2005) in this conference. Figure 8 shows the Science Payload Assembly excluding the secondary mirror tower.

The coronagraph starlight suppression assembly, all instruments, most of the payload electronics, and thermal control hardware mount to the PSS. Heaters maintain roomtemperature conditions. Heat pipes conduct heat from the electronics to a "warm" radiator and from the instrument detectors to a "cold" radiator. The entire lower portion

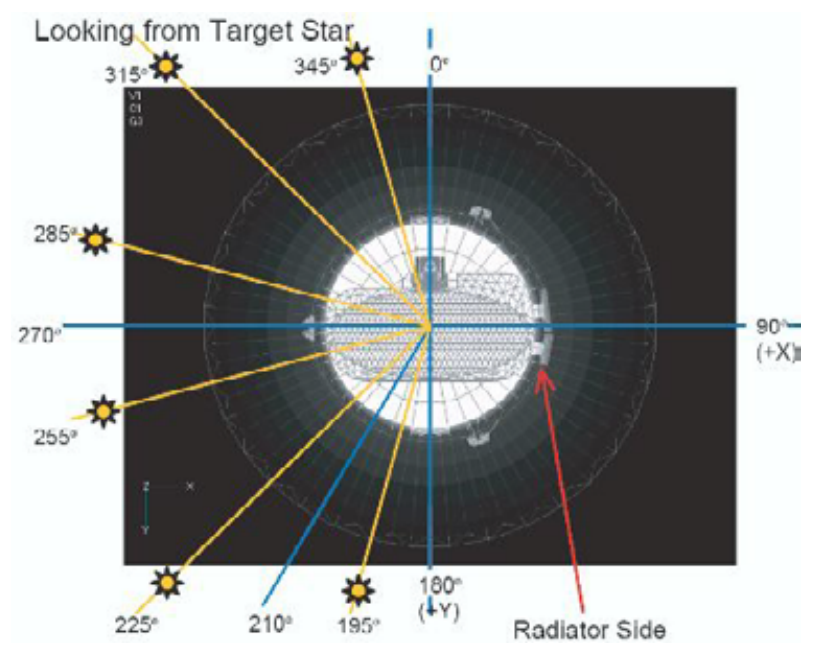

Figure 4. Observation angle nomenclature. 
of the payload is surrounded by a thermal enclosure mounted to the PSS. The PSS attaches to the spacecraft through three bi-pods. On the spacecraft side of the interface, at the end of each bi-pod, dynamic isolation is provided - either passive or active - both options were analyzed. The spacecraft carries thruster clusters, orbit maintenance fuel tanks, communications antennas, and reaction wheels. The sunshade mounts off of deployable tension truss, arms and booms. The solar panels and solar sail are also mounted on the spacecraft with deployable structures.

The final important part of the design is how it stows in launch configuration. The volume constraints of the launch shroud pose significant design limitations. Figure 9 shows the FB1 observatory stowed in its launch shroud. The Sun shade folds up and is stowed behind the payload around the spacecraft. The secondary tower folds along 4 joints to fit vertically alongside the primary mirror. The Solar Panels and Solar Sail fold up under the spacecraft, and the antenna arms fold alongside the spacecraft. Launch latches and launch support structure all release and fold away from the observatory upon deployment.

\section{Performance Analysis}

Several observational completeness models have been created that optimize the observing scenario to maximize planet discovery completeness. A paper (Brown 2005) earlier in this conference describes the approach.

Optical performance modeling is focused on developing propagation models that include all effects of the optical elements on the wavefront. Effects that are included are: diffraction, optical path differences, beam walk on non-perfect optical surfaces, spot

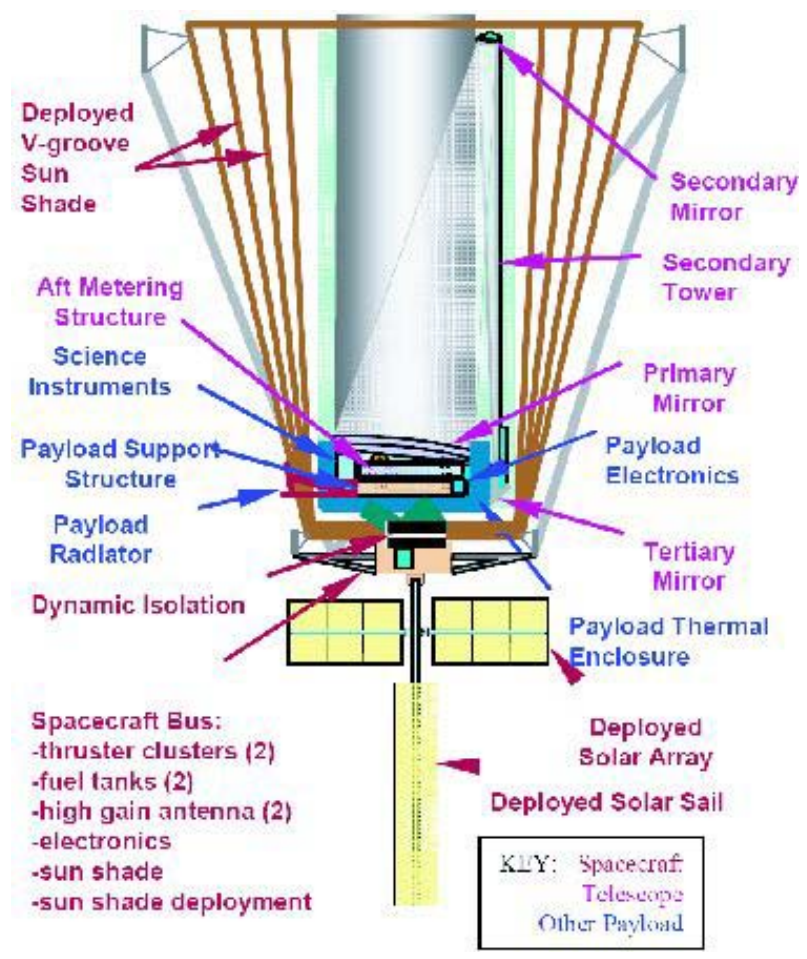

Figure 5. Flight Baseline 1 Schematic. 


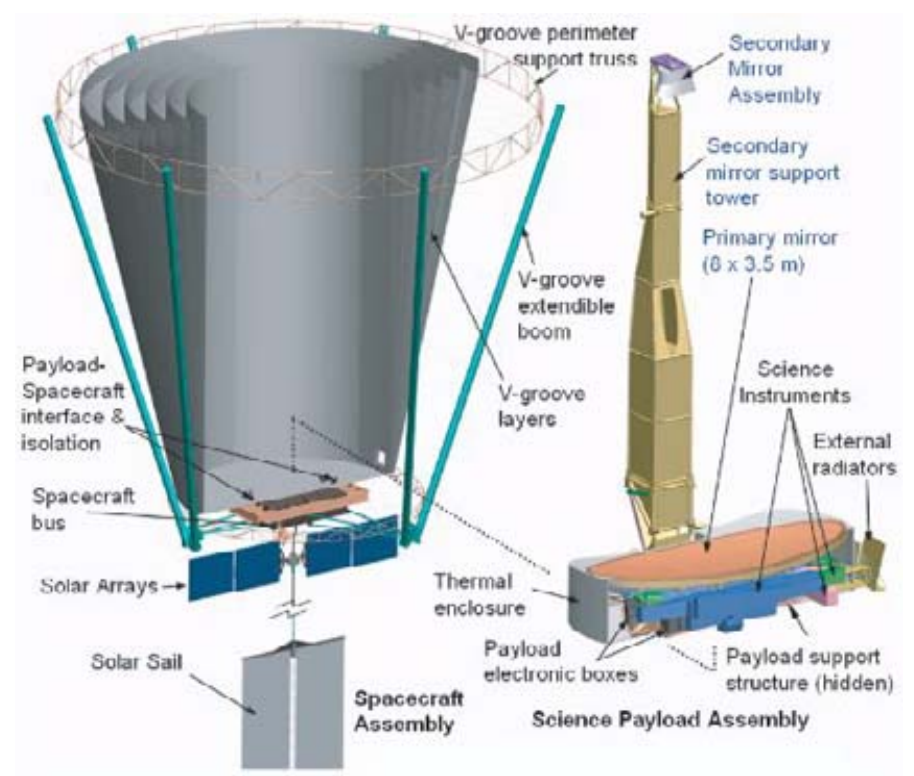

Figure 6. Observatory Design.

diagram centroid errors at occulting mask, jitter effects, cross-talk between polarization, phase, amplitude, and wavelength effects to develop a sensitivity matrix used to calculate contrast resulting from wavefront deformations. The modeling is on-going, but preliminary results are comparable to the error budget analysis that has been developed.

Thermal analysis has included modeling and comparing results from several thermal tools to determine tool accuracy, tool problems and develop solutions to permit achieving the accuracy needed for this observatory. Figure 10 shows the full thermal model using TMG software, and results of the temperature changes for the worst case dither scenario. Even in worst case conditions, the thermal changes meet the error budget allocation.

Structural models form the basis of the thermally induced wavefront aberration calculations and the pointing and control and jitter impact. Structural analysis predicts launch loads. Figure 11 shows the structural models, launch load results, and worst case thermally induced wavefront error. Figure 12 shows a high fidelity structural models of the primary mirror and the acoustic and static launch loads in the mirror. The stresses in the mirror are too high requiring a modification of the launch mounting scheme in the next design cycle.

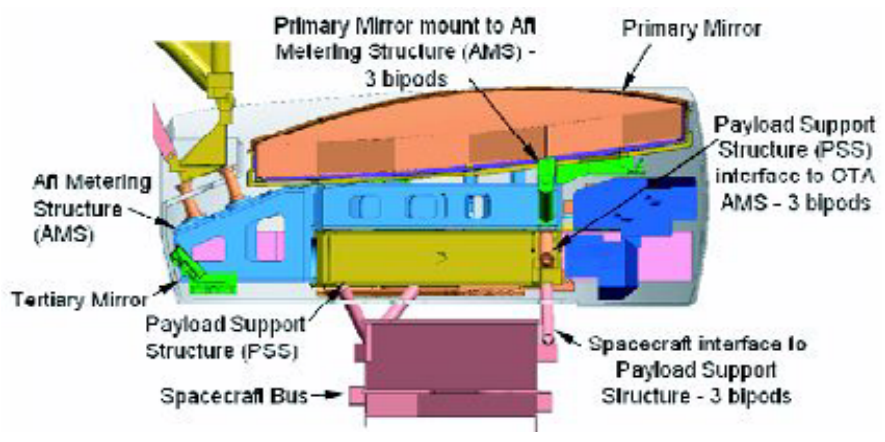

Figure 7. Science Payload Assembly (Ho). 


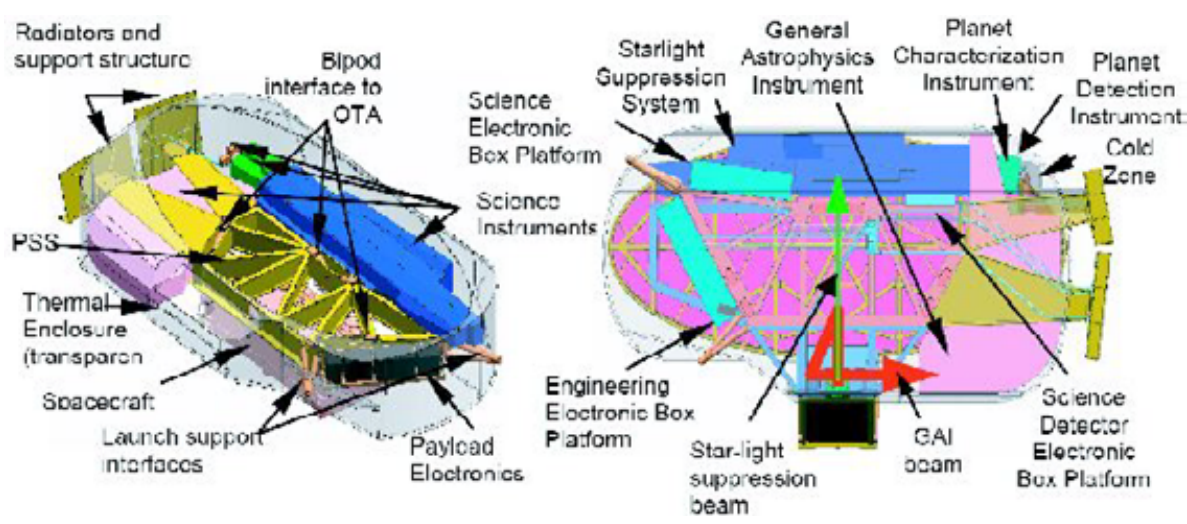

Figure 8. Science Payload excluding Telescope (T. Ho).

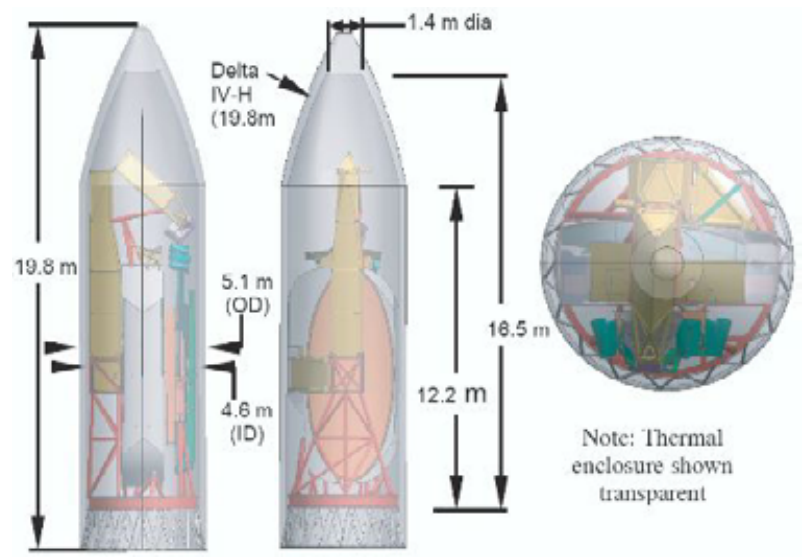

Figure 9. Flight Baseline 1 Launch Configuration (Ho).

The pointing control system concept is based on flight heritage sensors, reaction wheels, actuators, and control system. Two dynamic isolation systems were developed and analyzed for the suppression of reaction wheel disturbance: passive and active. The passive system currently does not meet requirements, but could be tuned with high likelihood of success. The active system meets requirements with large margin. Both designs are important for creating design space, and possibly developing redundancy. Figure 13 shows the worst case motion of the system optics as the reaction wheel speed changes. The top plots show translation and the bottom plots show rotation in three axes. The symbols at each optical element show the wheel speed at which the worst case response occurs. The passive system plot is on the left and the active system plot is on the right. The passive system optical responses are significantly over requirements for the SM, but within requirements for the downstream optics The passive system shows large angular excursions of the Fine Steering Mirror, produced by the control system as it keeps the target star positioned on the mask. The active system response is well below requirements in translation and rotation.

In conclusion, the design concept for Terrestrial Planet Finder Coronagraph continues to evolve with promising results. It appears that the environmental stability required for this project will be achievable. The modeling activities need to continue to develop to be sure light propagation effects are captured fully and understood so that the correct starlight suppression system can be maintained. There are some areas of the design that 


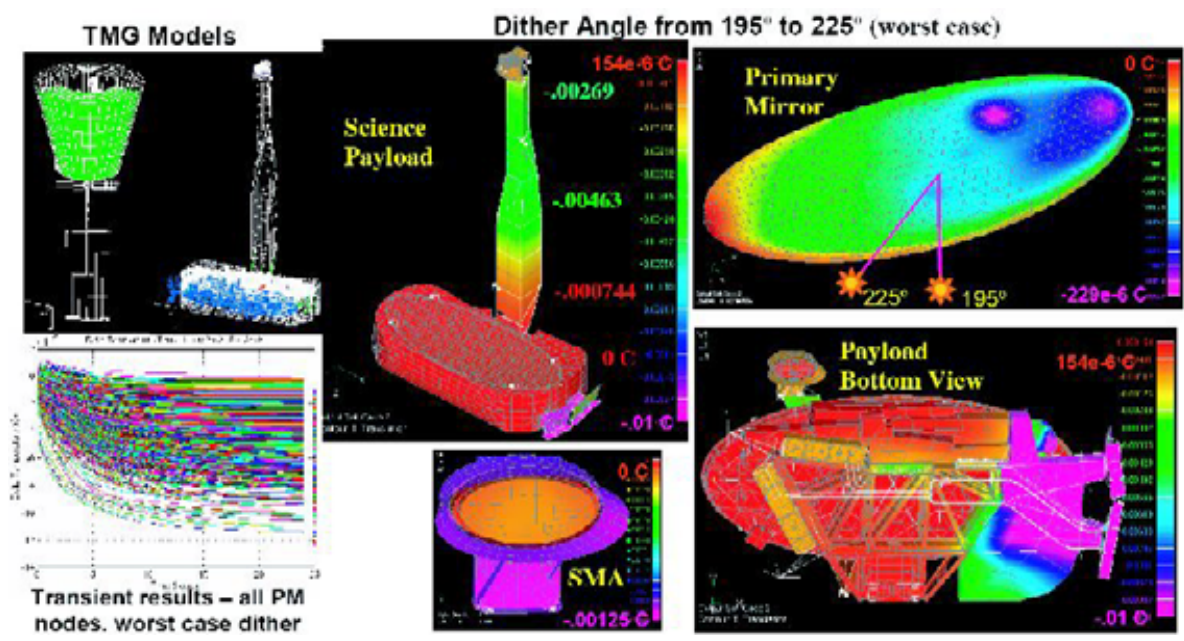

Figure 10. Thermal models and worst case dither temperature change results.
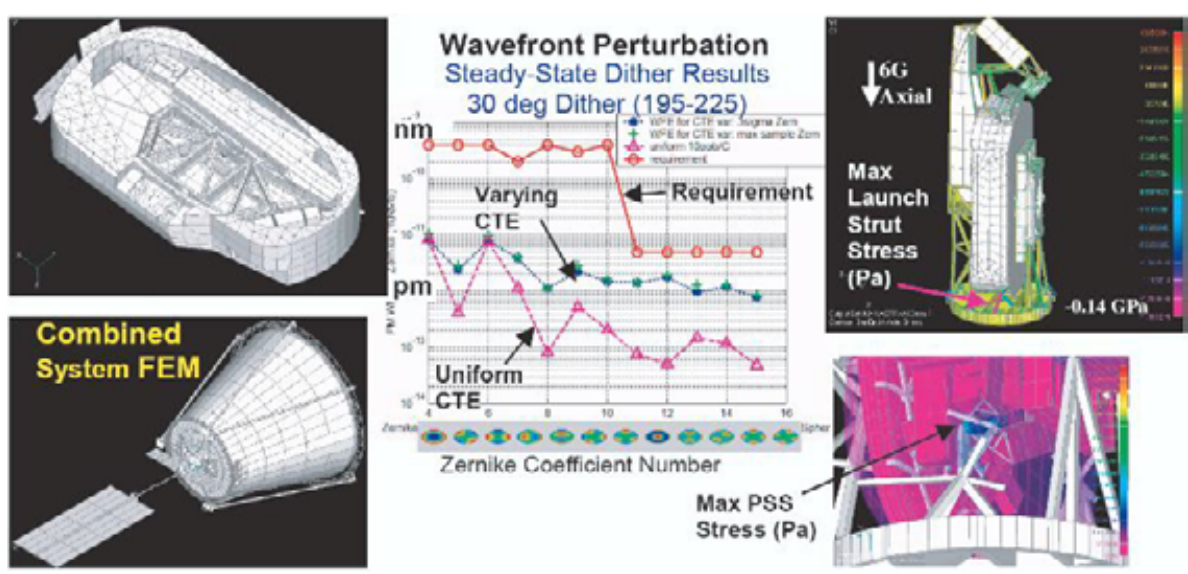

Figure 11. System structural model, Thermally-induced wavefront perturbation, and System Launch Stresses.

need to be addressed and improved, but they appear to be in the realm of engineering that can be accomplished.

\section{Acknowledgements}

I would like to thank the following people for their contributions to this paper: Marie Levine-West, Andy Kissil, Tim Ho, Eug Kwack, Phil Dumont, Dave Palacios, Jim Alexander, Sandra Irish, Chuck Engler, Lou Fantano, Alice Liu and Carl Blaurock.

This work was performed at the Jet Propulsion Laboratory, California Institute of Technology, under a contract with the National Aeronautics and Space Administration.

Reference herein to any specific commercial product, process or service by trade name, trademark, manufacturer, or otherwise, does not constitute or imply its endorsement by the United States Government or the Jet Propulsion Laboratory, California Institute of Technology. 


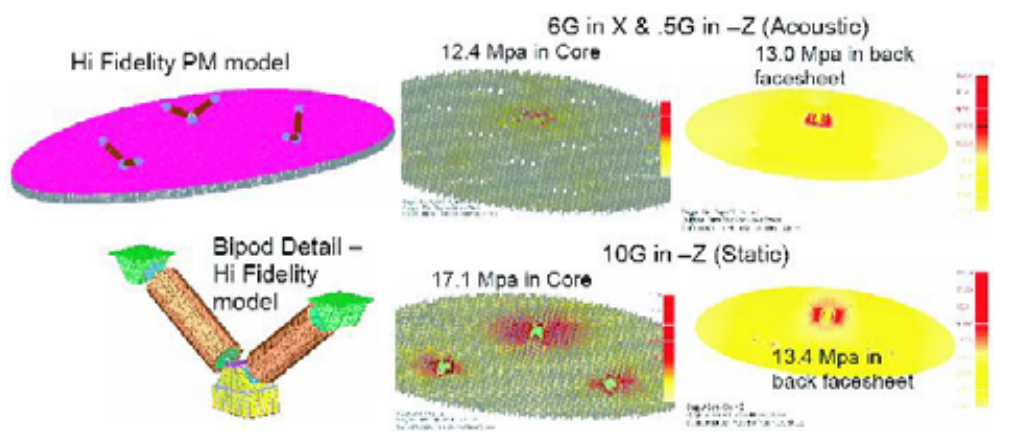

Figure 12. Hi Fidelity Primary Mirror model, detail of bipod model, and launch load results from both acoustic loading and static equivalent loading.
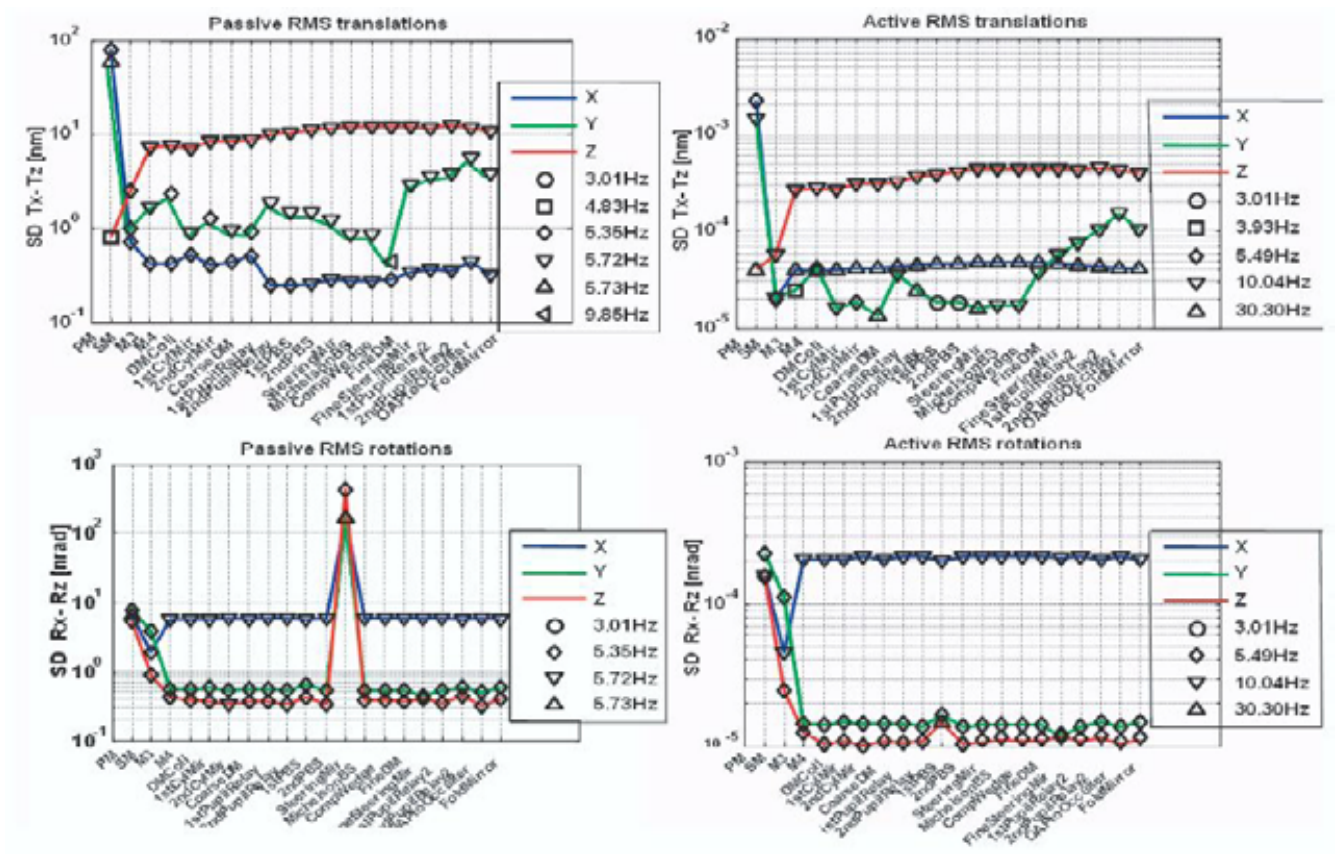

Figure 13. Dynamic response.

\section{References}

Trauger, J., "A case study in high contrast coronagraphy for planet discovery: the Eclipse concept and supporting laboratory experience," IAU 200, 6 Oct. 2005

Balasubramanian, K., "Occulting Focal Plane Masks for Terrestrial Planet Finder Coronagraph-Design, Fabrication, Simulations and Test Results," IAU 200, 4 Oct. 2005

Kuchner, M., "Eighth-order image masks for terrestrial planet finding," IAU 200, 6 Oct. 2005

Belikov, R., "Towards $10^{10}$ Contrast for NASA's Terrestrial Planet Finder Mission: Demonstration of High Contrast in a Shaped-Pupil Coronagraph," IAU 200, 6 Oct. 2005

Guyon, O., "The pupil remapping coronagraph," IAU 200, 6 Oct. 2005

Shao, M., "The Visible Nulling Coronagraph-Architecture Definition and Technology," IAU 200, 6 Oct. 2005

Shaklan, S., "Terrestrial planet finder coronagraph Instrument Design," IAU 200, 4 Oct. 2005

Ohl, R., "The Optical Telescope Assembly for the Terrestrial Planet Finder Coronagraph," IAU 200, 6 Oct. 2005

Brown, R., "Expectations in the Visible Domains," IAU 200, 4 Oct. 2005 